\title{
Magnetohydrodynamic Mixed Convection Around a Heat Conducting Horizontal Circular Cylinder in a Rectangular Lid-driven Cavity with Joule Heating
}

\author{
M. M. Rahman*, M. A. Alim, and M. K. Chowdhury \\ Department of Mathematics, Bangladesh University of Engineering and Technology, Dhaka-1000, \\ Bangladesh
}

Received 2 May 2009, accepted in final revised form 8 August 2009

\begin{abstract}
In the present paper, a study of magnetohydrodynamic (MHD) mixed convection around a heat conducting horizontal circular cylinder placed at the center of a rectangular cavity along with joule heating has been carried out. Steady state heat transfer by laminar mixed convection has been studied numerically by solving the equations of mass, momentum and energy to determine the fluid flow and heat transfer characteristics in the cavity as a function of Richardson number, Hartmann number and the cavity aspect ratio. The results are presented in the form of average Nusselt number at the heated surface; average fluid temperature in the cavity and temperature at the cylinder center for the range of Richardson number, Hartmann number and aspect ratio. The streamlines and isotherms are also presented. It is found that the streamlines, isotherms, average Nusselt number, average fluid temperature and dimensionless temperature at the cylinder center strongly depend on the Richardson number, Hartmann number and the cavity aspect ratio.
\end{abstract}

Keywords: Mixed convection; Finite element method; Cylinder diameter; Lid-driven cavity; Hartmann number.

(C) 2009 JSR Publications. ISSN: 2070-0237 (Print); 2070-0245 (Online). All rights reserved.

DOI: $10.3329 /$ jsr.v1i3.2597

J. Sci. Res. 1 (3), 461-472 (2009)

\section{Introduction}

The fundamental problem of combined free and forced convection heat transfer in a closed cavity has received considerable attention from researchers. Such a problem is usual grouped under lid driven cavity problems. This problem is often encountered in industrial process and in nature. The modelling and simulation of crystal growth, glass production, food processing and nuclear reactors are common examples of current industrial applications, while convective thermal currents associated with the flow structure occurring in the lakes and reservoirs are classically cited as a natural phenomenon.

\footnotetext{
*Corresponding author: mmustafizurrahman@math.buet.ac.bd
} 
Moallemi and Jang [1] studied numerically mixed convective flow in a bottom heated square lid-driven cavity. They investigated the effect of Prandtl number on the flow and heat transfer process. They found that the effects of buoyancy are more pronounced for higher values of Prandtl number, and also derived a correlation for the average Nusselt number in terms of the Prandtl number, Reynolds number and Richardson number. Iwatsu et al. [2] made numerical simulations for the flow of a viscous thermally stratified fluid in a square cavity. The flow was driven by both the top lid and buoyancy. They investigated the possibility of a resonance structure-giving rise to the intensification of the flows in the interior and associated augmentation of convective heat transport. Later on, Iwatsu et al. [3] and Iwatsu and Hyun [4] conducted respectively two- and three-dimensional numerical simulation of mixed convection in a square cavity heated from the top moving wall. Prasad and Koseff [5] reported experimental results for mixed convection in deep lid-driven cavities heated from below. They observed that the heat transfer was rather insensitive to the Richardson number. Aydin [6] conducted a numerical study to investigate the transport mechanism of laminar mixed convection in a shear- and buoyancy- driven cavity. Two orientations of thermal boundary conditions at the cavity walls were considered to simulate the aiding and opposing buoyancy mechanisms. Aydin and Yang [7] numerically studied mixed convection heat transfer in a two-dimensional square cavity having an aspect ratio of 1 . In their configuration the isothermal sidewalls of the cavity were moving downwards with uniform velocity while the top wall was adiabatic. A symmetrical isothermal heat source was placed at the other adiabatic bottom wall. They investigated the effects of Richardson number and the length of the heat source on the fluid flow and heat transfer. Mixed convection heat transfer in a two-dimensional rectangular cavity with constant heat flux from partially heated bottom wall while the isothermal sidewalls are moving in the vertical direction was numerically studied by Gau and Sharif [8]. Steady state two-dimensional mixed convection problem in a vertical twosided lid-driven differentially heated square cavity was investigated numerically by Oztop and Dagtekin [9]. They found that both Richardson number and direction of moving walls affect the fluid flow and the heat transfer in the cavity. Hossain and Gorla [10] investigated the effects of viscous dissipation on unsteady combined convective heat transfer to water near its density maximum in a rectangular cavity with isothermal wall. Two-dimensional flow in a two-sided lid-driven cavity containing a temperature gradient was investigated numerically by Luo and Yang [11].

In most studies found in the literature on mixed convection in a lid-driven cavity, no attention has been paid to the problem of MHD mixed convection in a lid-driven cavity containing a solid block. In the current investigation, the transport phenomena will be explored by utilizing several dimensionless parameters. These parameters are the Reynolds number, Hartmann number, Prandtl number, Richardson number Joule heating parameter and solid fluid thermal conductivity ratio. Here Richardson number is varied from 0.0 to 5.0 to simulate forced convection, mixed convection and free convection dominated flow in the cavity. The Hartmann number and cavity aspect ratio are considered from 0.0 to 50.0 and 0.5 to 2.0, respectively, while the values of Reynolds number, Prandtl number, Joule heating parameter and solid fluid thermal conductivity ratio are considered $100,0.71,1.0$ and 5.0, respectively. 


\section{Problem Definition}

The physical model under consideration and coordinates chosen are depicted in Fig. 1. A cartesian co-ordinate system is used with origin at the lower left corner of the computational domain. It is a two dimensional rectangular lid-driven cavity with a fixed amount of conducting solid material in the form of circular cylinder placed at the center of the cavity. The left wall of the cavity is allowed to move upward in its own plane at a constant velocity $U_{0}$ and to be kept at a constant temperature $T_{c}$. Horizontal walls of the cavity are insulated while the right vertical wall is assumed to be heated isothermally at a constant temperature $T_{h}$. In these figures, $H$ and $L$ are the height and length of the cavity respectively. Here the fluid is assumed to be electrically conducting, while walls of the cavity are considered to be electrically insulating. However, gravity acts in vertical directions and magnetic field is effective in the horizontal direction normal to the moving wall. The magnetic Re is assumed to be small so that the induced magnetic field is neglected and the Hall effects of magneto-hydrodynamics are to be negligible. All fluid physical properties are assumed to be constant except the density variation in the body force term of the momentum equation according to the Boussinesq approximation. In addition, the effect of Joule heating is considered, but pressure work and viscous dissipation are assumed to be negligible.

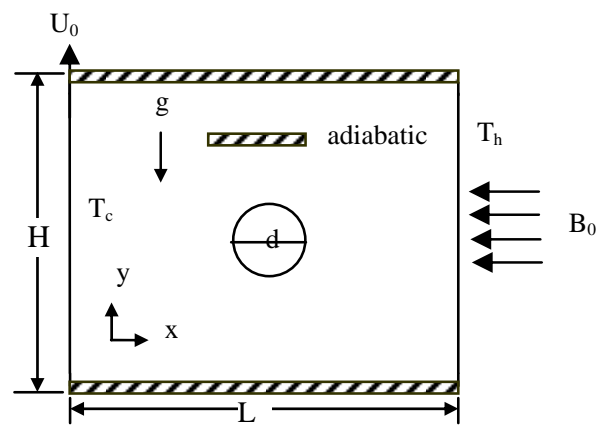

Fig. 1. Schematic of the problem with the domain and boundary conditions.

\section{Governing Equations with Boundary Conditions}

The electrically conducting fluids are assumed to be Newtonian fluids with constant fluid properties, except for the density in the buoyancy force term. Moreover, the fluid is considered to be laminar, incompressible, steady and two-dimensional. The electrically conducting fluids interact with an external horizontal uniform magnetic field of constant magnetic flux density $\mathrm{B}_{0}$. Assuming that the flow-induced magnetic field is very small compared to $\mathrm{B}_{0}$ and considering electrically insulated cavity walls. The electromagnetic force can be reduced to the damping factor $-B_{0} v$ ([15]), where $v$ is the vertical velocity component. Thus the Lorentz force depends only on the velocity component perpendicular 
to the magnetic field. Thus the governing equations for the problem can be expressed as follows:

$$
\begin{aligned}
& \frac{\partial U}{\partial X}+\frac{\partial V}{\partial Y}=0 \\
& U \frac{\partial U}{\partial X}+V \frac{\partial U}{\partial Y}=-\frac{\partial P}{\partial X}+\frac{1}{R e}\left(\frac{\partial^{2} U}{\partial X^{2}}+\frac{\partial^{2} U}{\partial Y^{2}}\right) \\
& U \frac{\partial V}{\partial X}+V \frac{\partial V}{\partial Y}=-\frac{\partial P}{\partial Y}+\frac{1}{R e}\left(\frac{\partial^{2} V}{\partial X^{2}}+\frac{\partial^{2} V}{\partial Y^{2}}\right)+R i \theta-\frac{H a^{2}}{R e} V \\
& U \frac{\partial \theta}{\partial X}+V \frac{\partial \theta}{\partial Y}=\frac{1}{\operatorname{RePr}}\left(\frac{\partial^{2} \theta}{\partial X^{2}}+\frac{\partial^{2} \theta}{\partial Y^{2}}\right)+J V^{2}
\end{aligned}
$$

For the solid cylinder, the energy equation in a dimensionless form becomes

$$
0=\frac{K}{\operatorname{Re} P r}\left(\frac{\partial^{2} \theta_{s}}{\partial X^{2}}+\frac{\partial^{2} \theta_{s}}{\partial Y^{2}}\right)
$$

The dimensionless variables used to make the governing Eqs. (1) -(5) are defined as

$$
X=\frac{x}{L}, Y=\frac{y}{L}, U=\frac{u}{U_{0}}, V=\frac{v}{U_{0}}, P=\frac{p}{\rho U_{0}^{2}}, D=\frac{d}{L}, \theta=\frac{\left(T-T_{c}\right)}{\left(T_{h}-T_{c}\right)}, \theta_{s}=\frac{\left(T_{s}-T_{c}\right)}{\left(T_{h}-T_{c}\right)}
$$

where

$$
\begin{aligned}
& R e=U_{0} L / v, G r=g \beta \Delta T L^{3} / v^{2}, H a^{2}=\sigma B_{0}^{2} L^{2} / \mu, \operatorname{Pr}=v / \alpha, R i=G r / R e^{2} \\
& J=\sigma B_{0}^{2} L U_{0} / \rho C_{p} \Delta T \text { and } K=k_{s} / k_{f}
\end{aligned}
$$

are the Reynolds number, Grashof number, square of the Hartmann number, Prandtl number, Richardson number Joule heating parameter and solid fluid thermal conductivity ratio respectively.

The dimensionless boundary conditions of the present problem under consideration can be written as follows:

At the left wall: $U=0, V=1, \theta=0$

At the heated right vertical wall: $U=0, V=0, \theta=1$

At the cylinder surface: $U=0, V=0$

At the top and bottom walls: $U=0, V=0, \frac{\partial \theta}{\partial N}=0$

At the fluid-solid interface: $\left(\frac{\partial \theta}{\partial N}\right)_{\text {fluid }}=K\left(\frac{\partial \theta_{S}}{\partial N}\right)_{\text {solid }}$

where $N$ is the non-dimensional distances either along $X$ or $Y$ direction acting normal to the surface. The average Nusselt number at the heated wall of the cavity based on 
the conduction contribution may be expressed as $N u=-\int_{0}^{1} \frac{\partial \theta}{\partial X} d Y$ and the average temperature in the cavity is defined as $\theta_{a v}=\int \theta d \bar{V} / \bar{V}$, where $\bar{V}$ is the cavity volume.

\section{Numerical Technique}

The numerical procedure used in this work is based on the Galerkin weighted residual method of finite element formulation. The application of this technique is well described by Taylor and Hood [16] and Dechaumphai [17]. In this method, the solution domain is discretized into finite element meshes, which are composed of triangular elements. Then the nonlinear governing partial differential equations i.e., mass, momentum and energy equations are transferred into a system of integral equations by applying Galerkin weighted residual method. The integration involved in each term of these equations is performed by using Gauss quadrature method. Then the nonlinear algebraic equations so obtained are modified by imposition of boundary conditions. These modified nonlinear equations are transferred into linear algebraic equations by using Newton's method. Finally, these linear equations are solved by using Triangular Factorization method. More details are available in Rahman et al. [13, 14]

\subsection{Grid refinement check}

In order to determine the proper grid size for this study, a grid independence test are conducted with $R e=100, R i=1.0, K=5.0, D=0.2, H a=10.0, J=1.0$ and $\operatorname{Pr}=0.71$. The following five types of mesh are considered for the grid independence study. These grid densities are 24427 nodes, 3774 elements; 29867 nodes, 4640 elements; 37192 nodes, 5814 elements; 38229 nodes, 5968 elements and 48073 nodes, 7524 elements. The extreme value of the average Nusselt number $\mathrm{Nu}$, that relates to the heat transfer rate of the heated surface and average and average temperature $\theta_{a v}$ of the fluid in the cavity are used as a sensitivity measure of the accuracy of the solution and are selected as the monitoring variables for the grid independence study. Table 1 shows the dependence of the quantities $N u$ and $\theta_{a v}$ on the grid size and the computational time. Considering both the accuracy of the numerical values and the computational time, the following calculations are performed with 38229 nodes and 5968 elements grid system.

Table 1 . Grid sensitivity check at $R e=100, R i=1.0, K=5.0, D=0.2, H a=10.0$, $J=1.0$ and $\operatorname{Pr}=0.71$.

\begin{tabular}{cccccc}
\hline $\begin{array}{c}\text { Nodes } \\
\text { (elements) }\end{array}$ & 24427 & 29867 & 37192 & 38229 & 48073 \\
\hline$N u$ & 1.022636 & $(4640)$ & $(5814)$ & $(5968)$ & $(7524)$ \\
$\theta_{a v}$ & 0.525566 & 0.525567 & 1.022650 & 1.022651 & 1.022651 \\
Time (s) & 226.265 & 292.594 & 3858.157 & 421.328 & 627.375 \\
\hline
\end{tabular}


Table 2. Comparison of the present data with those of Chamkha [12] for Ha.

\begin{tabular}{ccc}
\hline $\begin{array}{c}\text { Parameter } \\
\mathrm{Ha}\end{array}$ & $\begin{array}{c}\text { Present study } \\
\mathrm{Nu}\end{array}$ & $\begin{array}{c}\text { Chamkha [12] } \\
\mathrm{Nu}\end{array}$ \\
\hline 0.0 & 2.206915 & 2.2692 \\
10.0 & 2.113196 & 2.1050 \\
20.0 & 1.820612 & 1.6472 \\
50.0 & 1.18616 & 0.9164 \\
\hline
\end{tabular}

\subsection{Code validation}

For the purpose of code validation, the mixed convection problem in a lid driven cavity was tested for various values of $\mathrm{Ha}$ and $\mathrm{Gr}$. The calculated average Nusselt number at the hot wall for the test cases were compared with the values calculated by Chamkha [12]. As shown in Tables 2 and 3, the calculated average Nusselt number agrees well with the values calculated by Chamkha [12].

Table 3. Comparison of the present data with those of Chamkha [12] for Gr.

\begin{tabular}{ccc}
\hline Parameter & Present study & Chamkha [12] \\
$G r$ & $N u$ & $N u$ \\
\hline $10^{2}$ & 1.029805 & 0.9819 \\
$10^{3}$ & 1.105932 & 1.0554 \\
$10^{4}$ & 1.523059 & 1.4604 \\
$10^{5}$ & 2.462188 & 2.3620 \\
\hline
\end{tabular}

\section{Results and Discussion}

Results are presented for mixed convection inside an obstructed rectangular cavity where $R i$ has been varied from 0.0 to 5.0 by changing $G r$ while keeping $R e$ fixed at 100 . The Hartmann number and cavity aspect ratio are considered in the range from 0.0 to 50.0 and 0.5 to 2.0 , respectively.

\subsection{Effect of Hartmann number}

The influence of Hartmann number $(\mathrm{Ha})$ on the flow patterns at three different values of $R i$ is shown in Fig. 2, where $A R=1.0$ is kept fixed. In the absence of the magnetic field $(H a=0.0)$ and the natural convection effect $(R i=0.0)$, the fluid flow is characterized by a primary rotating uni-cellular vortex of the size of the cavity generated by the movement of the left wall. Again for $R i=0.0$ and the different higher values of $H a$ (10.0, 20.0 and 50.0), it is evident from these figures that the size of the vortex remain unchanged, but the core 


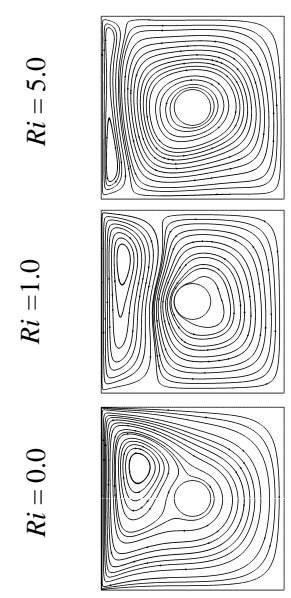

$H a=0.0$
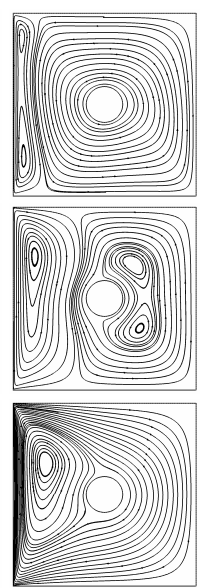

$H a=10.0$
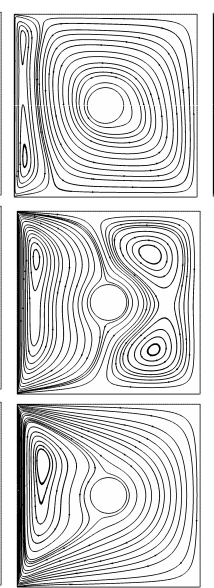

$H a=20.0$
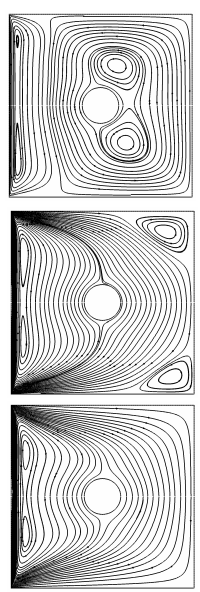

$H a=50.0$

Fig. 2. Streamlines for the different values of Hartmann number $(\mathrm{Ha})$ and Richardson number $(R i)$, while $A R=1.0$.

of the vortex shifted towards the left top corner of the cavity with increasing $\mathrm{Ha}$ up to 20.0 and the core divided into two parts located at the bottom and top corner near the left wall at the highest value of $\mathrm{Ha}=50.0$. When natural convection and forced convection become equally dominant, namely $R i=1.0$, the fluid flow is characterized by a clockwise rotating vortex generated by the movement of the left wall and two minor counter clockwise vortices generated by the buoyant force at the highest value of $\mathrm{Ha}=50.0$. As $\mathrm{Ha}$ decreases from 50.0 to 20.0, the minor counter clockwise vortices merge into a two cellular single one and become comparatively large, as a result the clockwise rotating vortex become smaller in size. Furthermore, the size of the clockwise vortex decreases and counter clockwise vortex increases with decreasing $H a$ at $R i=1.0$. This is because application of a transverse magnetic field has the tendency to slow down the movement of the buoyancy-induced flow in the cavity. Further when $R i=5.0$, the effect of natural convection is far more compared to the forced convection effect, consequently the size of the counter clockwise vortices at $R i=5.0$ are larger than that at $R i=1.0$. The corresponding effects of Hartmann number $\mathrm{Ha}$ on the isotherms is shown in the fig. 3 for the two aforesaid cases. From these figures it can be seen easily that the isotherms are almost parallel to the vertical walls for the highest value of $\mathrm{Ha}(\mathrm{Ha}=50.0)$ at the three values of $R i$, indicating that most of the heat transfer process is carried out by conduction. However, some deviations in the conduction dominated isothermal lines are initiated near the left top surface of the cavity for the value of $\mathrm{Ha}=20.0$ at $\mathrm{Ri}=0.0$. The distortion of the isotherms near the left top surface of the cavity increases quickly with decreasing values of $H a$ at $R i=0.0$. From these figures, it is also be seen that the isothermal lines are dominated by conduction and mixed convection heat transfer in the cavity for different $\mathrm{Ha}$ at $R i=1.0$. Although the isotherms are almost parallel to the vertical surface for the 
highest value of $\mathrm{Ha}$ at $\mathrm{Ri}=5.0$, but the isotherms are drastically changed with decreasing the values of $\mathrm{Ha}$ at $\mathrm{Ri}=5.0$. Moreover, the formation of the thermal boundary layer near the left cold wall is to be initiated here for the lower values of $\mathrm{Ha}$ at $\mathrm{Ri}=5.0$. This is owing to the dominating influence of the convective current in the cavity.
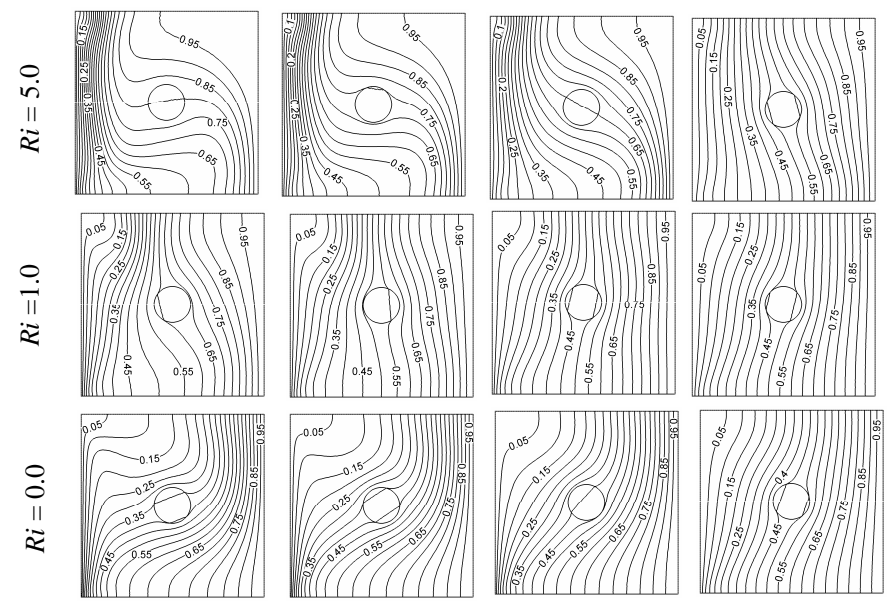

$$
H a=0.0
$$

$H a=10.0$

$H a=20.0$

$H a=50.0$

Fig. 3. Isotherms for the different values of Hartmann number $(\mathrm{Ha})$ and Richardson number $(R i)$, while $A R=1.0$.
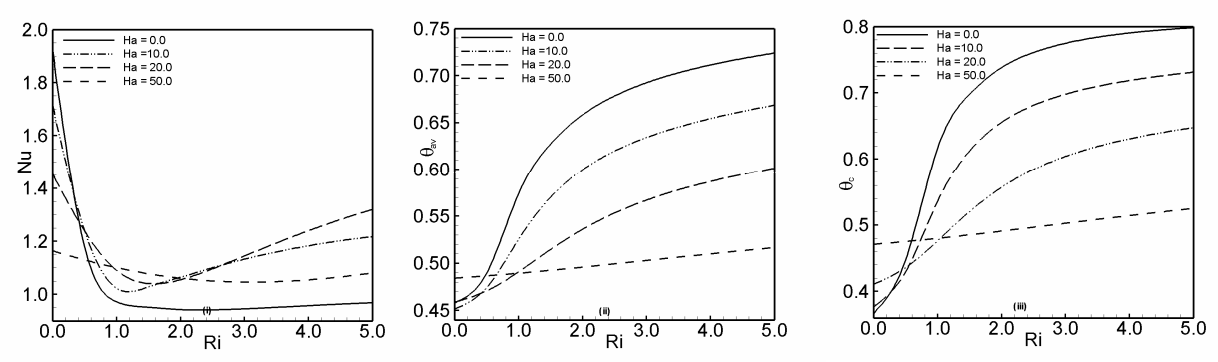

Fig. 4. Effect of Hartmann number ( $\mathrm{Ha}$ ) on (i) average Nusselt numbers, (ii) average fluid temperature and (iii) temperature at the cylinder center, while $A R=1.0$.

The effects of Hartmann number on average Nusselt number $(\mathrm{Nu})$ at the hot wall, average temperature $\left(\theta_{a v}\right)$ of the fluid in the cavity and the temperature $\left(\theta_{c}\right)$ at the cylinder center along with Richardson number is shown in the Fig. 4, while $A R=1.0$. From these figures, it is observed that the average Nusselt number $(\mathrm{Nu})$ goes down very rapidly with increasing $R i$ in the forced convection dominated region and goes up gradually with increasing $R i$ in the free convection dominated region for the lower values of $\mathrm{Ha}(0.0$, 10.0 and 20.0). But the average Nusselt number $(\mathrm{Nu})$ decreases mildly with increasing $\mathrm{Ri}$ for the highest value of $\mathrm{Ha}(\mathrm{Ha}=50.0)$. On the other hand, the values of $\mathrm{Nu}$ is the 
uppermost in the pure forced convection $(R i=0.0)$ at $H a=0.0$, in the mixed convection region $(R i=1.0)$ at $H a=50.0$ and in the natural convection dominated region at $\mathrm{Ha}=$ 20.0. However, the average temperature $\left(\theta_{a v}\right)$ of the fluid in the cavity and temperature $\left(\theta_{c}\right)$ at the cylinder center increases sharply with $R i$ for the lower values of $\mathrm{Ha}(0.0,10.0$ and 20.0) and increase very slowly with $R i$ at the highest value of $\mathrm{Ha}$.
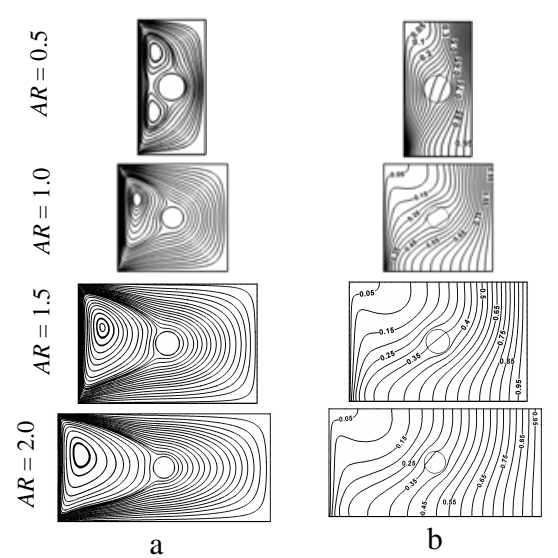

Fig. 5. (a) Streamlines and (b) Isotherms for various cavity aspect ratio and $\mathrm{Ha}=10.0$ at $\mathrm{Ri}=0.0$.
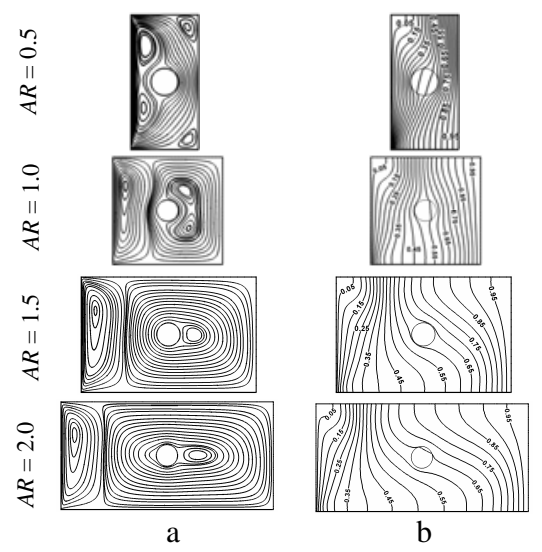

Fig. 6. (a) Streamlines and (b) Isotherms for various cavity aspect ratio and $\mathrm{Ha}=10.0$ at $\mathrm{Ri}$ $=1.0$.

\subsection{Effect of cavity aspect ratio}

The influence of the cavity aspect ratio on the flow and thermal fields in the cavity are shown in the Figs. 5 to 7 at three convective regimes. The aspect ratio is the ratio of the length $(L)$ and height $(H)$. At $R i=0.0$ and $A R=0.5$, it is seen that a two cellular vortex which we called primary vortex is generated due to the motion of the left wall. It is also seen from these figures that the vortex become uni-cellular and large in size with increasing $A R$ at fixed $R i=0.0$. Next at $R i=1.0$ and $A R=0.5$ it is observed that the primary vortex remain unchanged and two secondary vortices are developed at the top and bottom corner near the right wall due to the buoyancy effect. With the increase of $A R$ at $R i$ $=1.0$, it is seen that the size of the secondary vortex increases rapidly as a result, the size of the primary vortex decreases rapidly. Further, at $R i=5.0$ and all values of $A R(A R=$ $0.5,1.0,1.5$ and 2.0 ), it is seen that the secondary vortex spreads and thereby squeezes the primary vortex, indicating a sign of supremacy of natural convection in the cavity. Further For $R i=0.0$ and the four cavity aspect ratios $(A R=0.5,1.0,1.5$ and 2.0), the isothermal lines nearly follow the geometry of the right vertical surface and start to turn back towards the hot wall at the left top corner of the cavity due to the dominating influence of conduction and forced convection heat transfer. Now making a comparison of the isothermal lines for $R i=1.0$ and different $A R$ that of for $R i=0.0$ and different $A R$ no significant difference is found for the lower $A R$, but for higher $A R$ the isothermal lines 
start to turn back towards the cold wall in the upper part of the cavity. Moreover at $R i=$ 5.0 and lower $A R=0.5$ the isothermal lines are almost parallel to the left vertical and start to turn back towards the cold wall at the right top corner of the cavity due to the dominating influence of convective heat transfer. On the other hand, at $R i=5.0$ and higher values of $A R(1.0,1.5$ and 2.0) a significant convective distortion in the isothermal lines occurs due to the strong influence of the convective current as a result a concentrated thermal layer near the cold wall is developed.
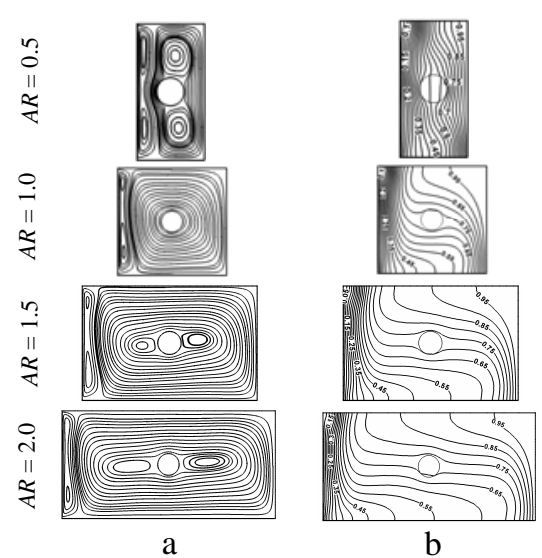

Fig. 7. (a) Streamlines and (b) Isotherms for various cavity aspect ratio and $\mathrm{Ha}=10.0$ at $R i=5.0$.
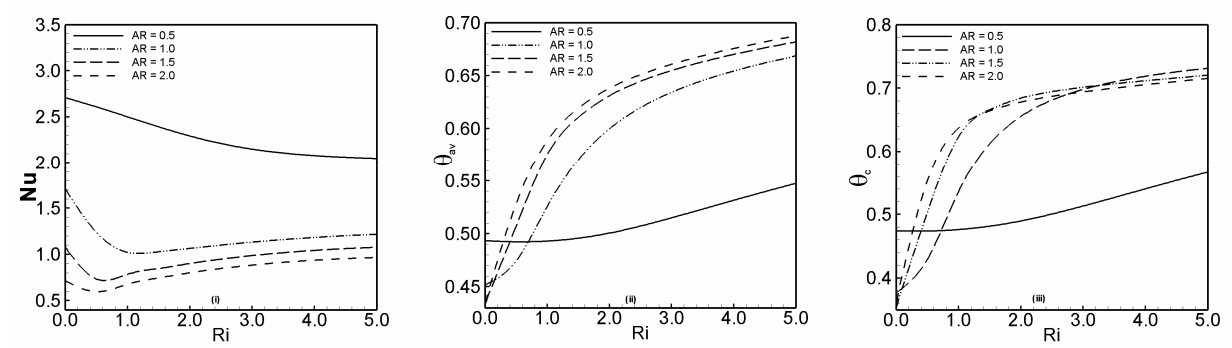

Fig. 8. Effect of cavity aspect ratio on (i) average Nusselt number, (ii) average fluid temperature and (iii) temperature at the cylinder center for different diameters of the cylinder, while $H a=10$.

The average Nusselt number $(\mathrm{Nu})$ at the heat source, average temperature $\left(\theta_{a v}\right)$ of the fluid in the cavity and the temperature $\left(\theta_{c}\right)$ at the cylinder center are plotted as a function of Richardson number in fig. 8 for the four values of $A R=0.5,1.01 .5$ and 2.0. For each higher $A R$, the $N u$-Ri profile shows two distinct zones depending on the Richardson number. The distribution of the average Nusselt number goes down sharply in the forced convection dominated region and goes up gradually in the free convection dominated 
region for the higher values of $A R(1.0,1.5$ and 2.0) with increasing $R i$. On the other hand, for the lowest value of $A R(A R=0.5)$, the average Nusselt number decreases monotonically with increasing Ri. However, maximum average Nusselt number is found for the low $A R=0.5$ at each $R i$ owing to the shortest distance between the hot and cold wall. From the figures 8 (ii) and 8 (iii), it is also seen that average temperature of the fluid in the cavity and the temperature at the cylinder center goes up sharply for the higher values of $A R$ (1.0, 1.5 and 2.0) and gradually for lowest $A R$ with increasing $R i$. In addition, the values of the average temperature of the fluid in the cavity and the temperature at the cylinder center are lower for the higher values of $A R$ in the forced convection dominated region, also for the lowest value of $A R$ in the free convection dominated region.

\section{Conclusion}

From the above study, the following conclusions have been drawn:

a. It is found that the flow behavior and the heat transfer characteristics inside the cavity are strongly depending upon the strength of the magnetic field. In the absence of the magnetic force, the convection-dominated zone is extended in the forced convection dominated region resulting better convective heat transfer performance. Increasing Hartmann number retards the fluid circulation causing the lower temperature gradients throughout the cavity in the forced convection dominated region. Therefore, major portion of the heat is transferred mainly by conduction. The inverse phenomena are observed in the free convection dominated area. However, lesser average fluid temperature and cylinder center temperature are observed for lower values of $\mathrm{Ha}(\mathrm{Ha}=10.0,20.0)$ at the forced convection dominated area and for $\mathrm{Ha}=50.0$ at the free convection dominated area.

b. Cavity aspect ratio has significant effects on the streamlines and isotherms distributions. Buoyancy-induced vortex in the streamlines and convective current in the isotherms increases with increasing aspect ratio of the cavity. Moreover, markedly different flow behaviors and heat transfer characteristics are observed among the three different flow regimes. On the other hand, average Nusselt number is always higher for lowest value of the cavity aspect ratio $A R(A R=0.5)$. The values of average temperature of the fluid in the cavity and temperature at the cylinder center are lower in the forced convection dominated region for the higher values of $A R$ and in the free convection dominated region for the lowest value of AR.

\section{Acknowledgements}

The authors like to express their gratitude to the Department of Mathematics, Bangladesh University of Engineering and Technology, for providing computer facility during this work. 


\section{References}

1. M. K. Moallemi and K. S. Jang, Int. J. Heat and Mass Transfer 35, 1881 (1992). doi:10.1016/0017-9310(92)90191-T

2. R. Iwatsu, J. M. Hyun, and K. Kuwahara, J. Fluids Engg. 114, 143 (1992).

3. R. Iwatsu, J. M. Hyun and K. Kuwahara, Int. J. of Heat and Mass Transfer 36, 1601 (1993). doi:10.1016/S0017-9310(05)80069-9

4. R. Iwatsu and J. M. Hyun, Int. J. of Heat and Mass Transfer 38, 3319 (1995). doi:10.1016/0017-9310(95)00080-S

5. A. K. Prasad and J. R. Koseff, Int. J. Heat and Fluid Flow 17, 460 (1996). doi:10.1016/0142-727X(96)00054-9

6. O. Aydin, Int. Commun. Heat Mass Transfer 26, 1019 (1999). doi:10.1016/S0735-1933(99)00091-3

7. O. Aydin and W. J. Yang, Numer. Heat Transfer (Part A) 37, 695 (2000). doi:10.1080/104077800274037

8. G. Guo and M. A. R. Sharif, Int. J. of Thermal Sciences 43, 465 (2004). doi:10.1016/j.ijthermalsci.2003.08.008

9. H. F. Oztop and I. Dagtekin, Int. J. of Heat and Mass Transfer 47, 1761 (2004). doi:10.1016/j.ijheatmasstransfer.2003.10.016

10. M. A. Hossain and R. S. R. Gorla, Int. J. of Numer. Methods for Heat and Fluid Flow 16 (1), 5 (2006). doi:10.1108/09615530610636928

11. W. J. Luo and R. J. Yang, Int. J. of Heat and Mass Transfer 50, 2394 (2007). doi:10.1016/j.ijheatmasstransfer.2006.10.025

12. A. J. Chamkha, Numer. Heat Transfer (Part A) 41, 529 (2002). doi:10.1080/104077802753570356

13. M. M. Rahman, M. A. Alim, and M. A. H. Mamun, Nonlinear Analysis: Modelling and Control 14 (2), 217 (2009)

14. M. M. Rahman, Ph.D. Thesis, Bangladesh University of Engineering and Technology, Dhaka, Bangladesh (2009).

15. K. R. Cramer, S. I. Pai, Magnetofluid Dynamics for Engineering and Applied Physicists, 1st Edition, (McGraw-Hill, New York, 1974)

16. C. Taylor and P. Hood, Computer and Fluids 1, 73 (1973). doi:10.1016/0045-7930(73)90027-3

17. P. Dechaumphai, Finite Element Method in Engineering, 2nd Edition, (Chulalongkorn University Press, Bangkok, 1999). 